\title{
A brief review of photo-orthotropic elasticity theories
}

\author{
K RAMESH* and N TIWARI \\ Department of Mechanical Engineering, Indian Instutute $\$ 17 \mathrm{cech}$ \\ Kanpur 208016 , India
}

MS received 22 September 1992; revised 12 April 1993

\begin{abstract}
The development of the theory of birefringence composites has proceeded mainly along the following directhum, strain models, analogy, integrated photoelasticity and tensortal mats birefringence. All these studies have concluded that three independents

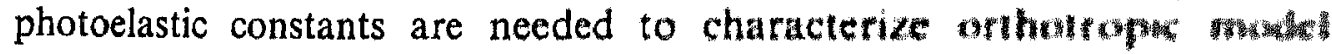
materials. In the literature, there has not been uniformily of nowatuan unest in the representations of the results.

In this paper, using uniform notation, existing photomorthotuspes chas

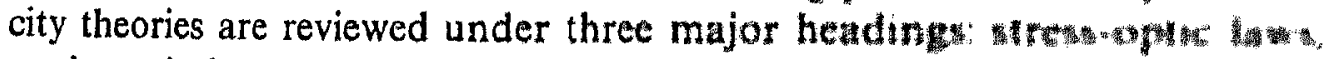
strain-optic laws and approximate strain-optic laws. The interrets ships between stress-optic and strain-optic coefficients are brough

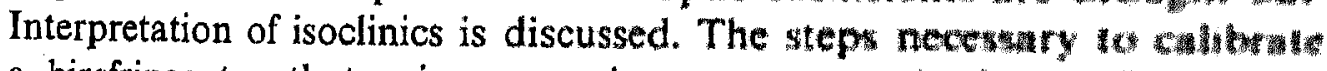
a birefringent orthotropic composite are summarized and hnally influence of residual birefringence is also discussed.
\end{abstract}

Keywords. Orthotropic composites; photoelasticity: photomorthorropect elasticity; stress-optic law; strain-optic law.

\section{Introduction}

Composite materials have established themselves as strong candidates for importan structural applications. For their efficient utilization, complete stress analy reliable failure criteria are essential. Analytical methods tend to become difficuit fos composites due to material anisotropy, and multiplicity of failure modew Convequathy. experimental methods of stress analysis and failure analysis are very useful fort composites (Whitney et al 1984).

Among various experimental methods, optical methods have the advantage of yielding full-field quantitative results. In recent years there have been uninificant efforts to extend photoelastic methods of stress analysis for application involwing composites. One approach outlined by Dally \& Alfirevich (1963), is to employ woutropte birefringent coatings bonded to the composite. In this case, the strain feld w deterwaswed

*For correspondence 
from the coating using a reflection polariscope together with conventional methods of photoelastic analysis established for isotropic materials.

The second approach, initiated by Pih \& Knight (1969) involves the use of a transparent birefringent model of the composite with anisotropic elastic and optical properties.

Though the first approach is simple in its application, there are several difficulties associated with it. Pipes \& Dally (1972) conducted detailed analysis near free edges of composite materials and pointed out the limitations of the method due to the mismatch of the Poisson's ratios of the coating and the substrate. Further, the birefringent coating method is not suitable for studies on the dynamic fracture of composites since the coating reinforces the laminate and arrests crack growth.

On the other hand, development of transmission photoelastic techniques for composites is the right choice for studying dynamic crack propagation in composites. One of the important requirements for this technique is the development of appropriate model materials. This has been addressed by several investigators (Pih \& Knight 1969; Agarwal \& Chaturvedi 1970; Sampson 1970; Dally \& Prabhakaran 1971; Prabhakaran 1980b; Tiwari 1990; Agarwal et al 1991).

The second difficulty is the interpretation of the optical response. In the case of isotropic model materials, the optical response can be quantitativcly related to the differences in principal stresses using a single stress-optic coefficient.

In the case of a transparent composite laminate, the state of stress at each point along a light beam through the model thickness is different. The optical response observed in a polariscope is due to the integral effect of the stress field through the thickness of the model. It has been observed experimentally that the orientation of fibres influences the photoelastic response, and hence, a single photoelastic constant is not sufficient to characterize the model material. In the past two decades research work in this area has been primarily done for the determination of calibration constants for unidirectional composites.

In the following paragraphs, using uniform notation, various existing photoorthotropic eiasticity theories are briefly reviewed. Interrelationships between stressoptic and strain-optic coefficients are brought out. Interpretation of isoclinics is discussed. The steps required to characterize transparent orthotropic composite laminates are summarized and finally the influence of residual birefringence is also discussed. A detailed review can be found in the ARDB report of Ramesh \& Tiwari (1991).

\section{Stress-optic laws}

Though Pih \& Knight (1969) were the pioneers in analysing transparent composites using the principle of stress proportioning, their results are far from satisfactory due to inaccuracies in their stress-strain model. Sampson (1970) developed the concept of "Mohr's circle of birefringence" by simple analogy and arrived at the following relation for fringe order $N$,

$$
N=\left[\left\{\left(\sigma_{L} / f_{L}\right)-\left(\sigma_{T} / f_{T}\right)\right\}^{2}+\left\{\left(2 \tau_{L T} / f_{L T}\right)\right\}^{2}\right]^{1 / 2} \cdot t
$$

Let the principal stresses be $(p, q)$ and the principal stress $p$ be at an angle $\alpha$ to the $L$ axis, then (1) becomes 


$$
\begin{gathered}
N=\left[\left\{\left[\cos ^{2} \alpha+(q / p) \cdot \sin ^{2} \alpha\right]-\left[\sin ^{2} \alpha+(q / p) \cdot \cos ^{2} \alpha\right]\left(f_{L} / f_{T}\right)\right\}^{2}+\right. \\
\left.[1-(q / p)]^{2} \cdot\left[\sin 2 \alpha \cdot\left(f_{L} / f_{L T}\right)\right]^{2}\right]^{1 / 2} \cdot\left(t \cdot p / f_{L}\right) .
\end{gathered}
$$

For a uniaxial stress-field (i.e. $q=0$ ),

$$
N=\left[\left\{\left(\cos ^{2} \alpha / f_{L}\right)-\left(\sin ^{2} \alpha / f_{T}\right)\right\}^{2}+\left(\sin ^{2} 2 \alpha / f_{L T}^{2}\right)\right]^{1 / 2} t \cdot p
$$

It has been pointed out earlier that the fibre-orientation angle influences the fringe order. In other words the material fringe value is a function of fibre-orientation angle. If $f_{\alpha}$ is defined as $t \cdot p / N$ then the above equation directly gives the value of $f_{\alpha}$. Extending the analogy of Mohr's circle, Sampson (1970) obtained the optical isoclinic angle $\phi$ as

$$
\tan 2 \phi=\left(2 \tau_{L T} / f_{L T}\right) /\left[\left(\sigma_{L} / f_{L}\right)-\left(\sigma_{T} / f_{T}\right)\right]
$$

In terms of the fibre orientation angle for a uniaxial stress field,

$$
\tan 2 \phi=\left(f_{L} \cdot \sin 2 \alpha\right) /\left[f_{L T} \cdot\left\{\cos ^{2} \alpha-\left(f_{L} / f_{T}\right) \cdot \sin ^{2} \alpha\right\}\right],
$$

and for a general biaxial stress field,

$$
\begin{aligned}
\tan 2 \phi=\left[f_{L} \cdot(1-q / p) \cdot \sin 2 \alpha\right] /\left[f_{L T} \cdot\right. & \left\{\cos ^{2} \alpha+q \cdot \sin ^{2} \alpha / p\right. \\
& \left.\left.-\left(f_{L} / f_{T}\right) \cdot\left(\sin ^{2} \alpha+q \cdot \cos ^{2} \alpha / p\right)\right\}\right] .
\end{aligned}
$$

Using the analogy approach, Sampson (1970) could show that three photoelastic constants $\left(f_{L}, f_{T}\right.$ and $\left.f_{L T}\right)$ are required to characterize orthotropic materials. $\mathrm{He}$ supported his theory by limited experimental results. No mathematical proof was provided by him to support his theory. However, all later investigators, proceeding from various standpoints substantiated his results.

Dally \& Prabhakaran (1971) also predicted three fundamental photoelastic constants. Their important contribution is that they evaluated these constants as functions of the elastic and optical properties of the constituents of the composites. While formulating their model, they assumed that the total retardation introduced by the laminate is the algebraic sum of retardation introduced by fibre and matrix phases. Using the principle of stress proportioning and the results of Greszczuk (1966), they showed that the accuracy of the results is greatly influenced by the accuracy with which the stresses in the constituents can be evaluated. Their experimental results agreed closely with the stress-optic law formulated by Sampson (1970) (figure 1). However, they did not discuss the interpretation of isoclinics.

Bert (1972) introduced the application of tensorial-relations in the formulation of the stress-optic laws by adapting the work of Bhagavantham (1966) on the crystal theory. In this theory, stress and birefringence were shown as second-order tensors so that the photoelastic properties, tensor relating stress and birefringence must be of fourth order. Symmetry of an aligned fibrous composite was assumed to be representable by an orthorhombic crystal. The equations presented were in a form not practically suitable for solving general plane-stress problems. No experimental data were presented in their paper.

Cernosek (1975), using the principle of integrated photoelasticity analysed the orthotropic composite and arrived at the same results as Sampson (1970) and Bert (1972). The approach of integrated photoelasticity explicitly takes care of the variation 


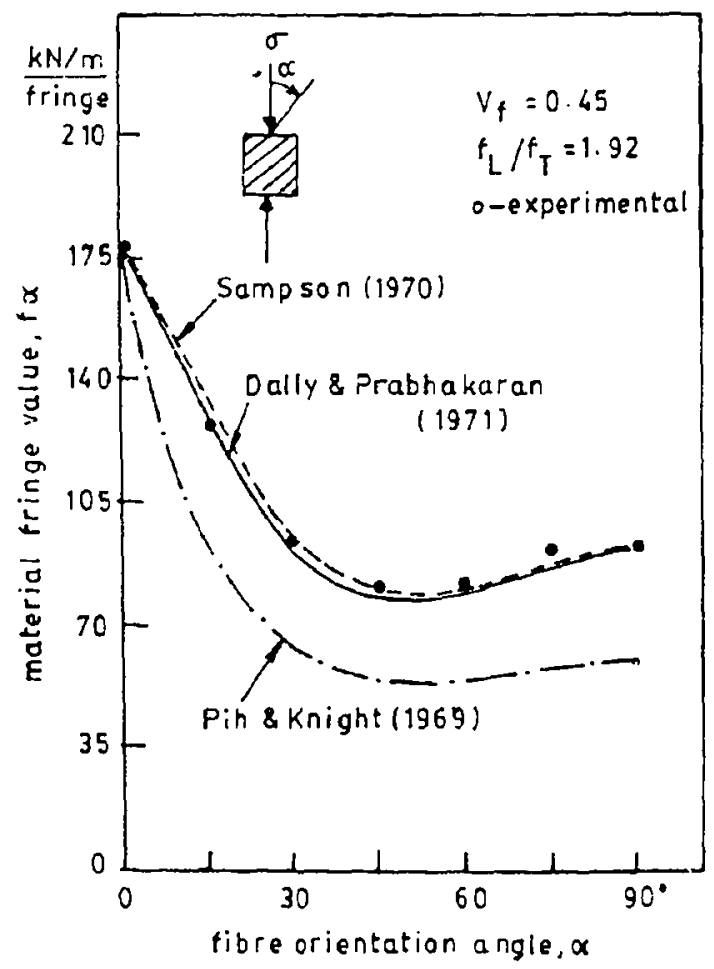

Figure 1. Material-fringe value $f_{x}$ for a unidirectional laminate as a function of fibre orientation angle (from Dally \& Prabhakaran 1971).

of the principal stress direction and magnitude from point to point along the path of light. Using the principle of "optical equivalence" (Aben 1966), even for the most general case of principal stress variation both in magnitude and direction along the path of light, the net optical effect could be reduced to a combination of a retarder and a rotator. Cernosek (1975) observed that for the case of orthotropic composites, the optical effect could be represented by a retarder alone with the following retardation,

$$
2 \delta_{0}=2 n\left[\delta_{f}^{2}+\delta_{m}^{2}+2 \delta_{f} \cdot \delta_{m} \cdot \cos 2 \Omega\right]^{1 / 2}
$$

He obtained the orientation of the retarder to be the same as that given by (5). Using Dally and Prabhakaran's (1971) stress-strain model, he showed that (7) could be reduced to (3). Thus he established a similarity between Sampson's (1970) phenomenological theory and Prabhakaran's stress-proportioning concept. Further, he showed that the expression of $f_{\alpha}$ developed by Dally \& Prabhakaran (1971) is exact only when $\alpha=0^{\circ}$ or $90^{\circ}$. In contrast to this, Sampson's (1970) theory is valid for any value of $\alpha$. Though the expression for $f_{\alpha}$ as developed by Dally \& Prabhakaran (1971) is approximate, the expression for $f_{L}$ and $f_{T}$ in terms of the parameters of the composite's constituents is exact since by definition $\alpha=0^{\circ}$ or $90^{\circ}$ in such cases.

Knight \& Pih (1976) using the Bhagavantham (1966) theory derived a general stress-optic law and then simplified it to the two-dimensional case. Their formulation was general and also accounts for initial birefringence. Their simplified equations are of the same form as those of Sampson (1970) and Bert (1972).

Equations (2), (3), (5) and (6) directly show the influence of fibre orientation. While using these equations, it is to be remembered that the angle $x$ is the orientation of principal stress ' $p$ ' with respect to the ' $L$ ' axis of the composite. Through explicitly bringing in the equations, the influence of fibre orientation angle has led to certain simplifications in the case of strain optic laws; (1) and (4) are recommended for general use because of their generality and simplicity. 


\section{Strain-optic laws}

The reduced influence of mechanical anisotropy on the strain fringe value, compared to its effect on the stress-fringe value, has been recognized by Horridge (1955). Hayashi (1962), Grakh \& Mozhenskaya (1971) and Netrebko (1971). Pipes \& Rose (1974) developed a strain-optic law involving only one photoclastic constint. However, their results are applicable only to unidirectional composites having a very low fibre content. Following the approach of Sampson (1970), Prabhakaran (1975) developed a strain-optic law based on the concept of Mohr's circle of hirefringence and introduced three material strain-fringe values, $f_{\varepsilon L}, f_{k T}$ and $f_{t, 1}$. These are related $n$ the observed fringe order in the model material by the relation,

$$
N=\left[\left\{\left(\varepsilon_{L} / f_{\varepsilon L}\right)-\left(\varepsilon_{T} / f_{\varepsilon T}\right)\right\}^{2}+\left\{\left(\hat{\gamma}_{L T T} / f_{\varepsilon L T}\right)\right\}^{2}\right]^{1 / 2} \cdot t .
$$

The isoclinic angle is given by

$$
\tan 2 \phi=\left(\gamma_{L T} / f_{s L T}\right) /\left(\varepsilon_{L L} / f_{s, L}-\varepsilon_{T} / f_{L I T}\right)
$$

Agarwal \& Chaturvedi (1982) derived an exact strain-optic law for orthotropic materials using Pockel's (1906) theory of crystalline photoelasticity. Their approach is similar to the method followed by Knight \& Pih (1976) for stress-optic liws. Chaturvedi (1982) pointed out that $\delta_{3}$ also contributes to the photoclastic cffect. The final form of the equations obtained by Prabhakaran (1975) and Agarwal \& Chaturvedi (1982) are identical. They also concluded that three material strain fringe values are required for the total characterization of the composite model material.

\section{Approximate strain-optic laws}

The proponents of strain-optic laws did not stop at their exact theories but also devised ways to develop approximate strain-optic laws for better and practical utiliation. Important contributions in this direction were made by Agarwal and his coworkers (Jha 1980; Agarwal \& Chaturvedi 1982; Agarwal 1983). Prabhakaran (1975) also proposed an approximate strain-optic law. In this approximate law he defined a single strain-optic coefficient, whose value was taken as the algebraic mean of the three strain-optic coefficients. Since the quantities under consideration are tensorial in nature, algebraic addition is not strictly correct.

Agarwal \& Chaturvedi (1982) proposed an approximate strain-optic law, in which the square of the error between the predictions of exact and approximate laws was minimized. However, the accuracy of the approximate strain-optic law was found to vary with the fibre orientation angle and also with the volume fraction of the fibres. Later, Jha (1980) and Agarwal (1983) modified the approximate law of Agarwal \& Chaturvedi (1982) slightly and proposed a new approximate strain optic law which can be written as

$$
\begin{aligned}
& \left.N=\left[\left(\varepsilon_{p}-\varepsilon_{q}\right) / f_{\ell L}\right]\left[\cos ^{2} 2 \beta_{c}+\left(f_{e L} / f_{\ell L T}\right)^{2} \cdot \sin ^{2} 2 \beta_{c}\right)\right]^{1 / 2} \cdot t, \\
& \phi=1 / 2 \cdot \tan ^{-1}\left\{\left[f_{\varepsilon L} / f_{\varepsilon L T}\right] \cdot \tan 2 \beta_{c}\right\} .
\end{aligned}
$$

This law is exact for a balanced composite and approximate for a general one. The predictions were found to be in good agreement with experimental results. 


\section{Interrelationships between stress and strain optic coefficients}

Using Mohr's circle of birefringence, Prabhakaran (1979) has obtained the relationships between material strain-fringe and stress-fringe values as follows

$$
\begin{aligned}
f_{\varepsilon L} & =\left(1-v_{L T} \cdot v_{T L}\right) /\left[\left(E_{L} / f_{L}\right)-\left(E_{T} \cdot v_{L T} / f_{T}\right)\right], \\
f_{\varepsilon T} & =\left(1-v_{L T} \cdot v_{T L}\right) /\left[\left(E_{T} / f_{T}\right)-\left(E_{L} \cdot v_{T L} / f_{L}\right)\right], \\
f_{\varepsilon L T} & =f_{L T} / 2 G_{L T} .
\end{aligned}
$$

Inverting the above set of equations one obtains

$$
\begin{aligned}
& 1 / f_{L}=\left[1 /\left(E_{L} \cdot f_{\varepsilon L}\right)\right]+v_{L T} /\left(E_{L} \cdot f_{\varepsilon T}\right), \\
& 1 / f_{T}=\left[1 /\left(E_{T} \cdot f_{\varepsilon T}\right)\right]+v_{T L} /\left(E_{T} \cdot f_{\varepsilon L}\right), \\
& 1 / f_{L T}=1 /\left(2 G_{L T} \cdot f_{\varepsilon L T}\right) .
\end{aligned}
$$

Doyle (1978), starting from the premise that optical and mechanical constitutive relations do not necessarily have similar forms, showed initially that two constants are sufficient to get the optical response of the composites. This suggests that there must be an interrelationship between $f_{L}, f_{T}$ and $f_{L T}$. In a discussion of the above paper, Prabhakaran (1980a) explicitly expressed the interrelationship as

$$
\frac{1}{f_{L T}}=\frac{1}{f_{L}}-\left[\frac{1}{f_{L}}-\frac{1}{f_{T}}\right] \cdot\left[\frac{1+v_{L T}}{E_{L_{L}}}-\frac{1}{2 G_{L T}}\right] /\left[\frac{1}{E_{L}}-\frac{1}{E_{T}}\right] .
$$

In a later paper, Doyle (1980) emphasized that two constants are insufficient if the volume fraction of the fibre in the composite exceeds by $6 \%$. Thus, (18) holds good if the volume fraction of fibre in the composite is less than $6 \%$. For cases when fibre content in composite exceeds this limit, knowledge of three independent constants is essential for proper characterization of the model material.

It is interesting to note that the stress-fringe values can be related to the properties of the constituents of the composite. Dally \& Prabhakaran (1971) obtained one such set of relations for $f_{L}, f_{T}$ and $f_{L T}$.

\section{Interpretation of isoclinics}

Pih \& Knight (1969) realized that the isoclinics in orthotropic models do not give the principal stress directions of the composite. Although Sampson (1970) found strong support from the isochromatic results of Pih \& Knight (1969) for his stressoptic law based on analogy, he could not show a similar agreement between his predictions and the isoclinic results of Pih \& Knight (1969). Pipes \& Dalley (1973) pointed out that the experimenal isoclinic results of Pih \& Knight (1969) could not be deemed reliable due to end-effects; they measured the isoclinic parameters for two fibre orientations and concluded that the isoclinic parameter is influenced by the stress level (due to the residual effects caused by matrix shrinkage) and that the isoclinics give neither the principal stress directions nor the principal strain directions. They revived the possibility that Sampson's (1970) concept of the Mohr circle of birefringence could predict the isoclinic parameters but did not substantiate this 
possibility. Pipes \& Rose (1974) hypothesized that the isoclinic parameter gives the principal strain direction.

Prabhakaran (1976), working on the premise that Sampson (1970) was unable to verify his isoclinic predictions because of the absence of reliable experimental results, measured the isoclinic parameters in uniaxial as well as biaxial stress-fields. For a uniaxial stress field, (5) relates the optical isoclinic with the fibre orientation as shown in figure 2. It is seen that the predictions based on the concept of Mohr's circle of birefringence (i.e. orthotropic theory using (5)) agree with the experimental results very well. Various other angles, such as the principal stress direction $\left(\Theta_{c}\right)$, principal strain direction $\left(\beta_{c}\right)$ of the composite, principal stress direction of fibre $\left(\Theta_{f}\right)$ and matrix $\left(\Theta_{m}\right)$ are also shown in figure 2 . It is clear from the graph that the optical isoclinic parameter is a closer approximation to the principal strain angle than to the principal stress angle. It may be noted that in figure 2, the angles are measured with the $L$-axis as reference.

The isoclinic parameter has been investigated in biaxial stress-fields (Prabhakaran 1976) also. A circular disk, unidirectionally reinforced, was diametrically compressed at different orientations. The optical isoclinic parameter at the centre of the disk was measured and the strains at the centre were also measured by a strain gauge rosette. The influence of the residual birefringence can be seen in figure 3 . At sufficiently high loads, the measured isoclinic parameter could be considered as due to the applied load.

In figure 4 , the optical isoclinic parameter $(\phi)$, using $(6)$, is shown as a function of the fibre-orientation angle $\alpha$. Also shown in the figure are the principal stress angle (physical isoclinic parameter) and the principal strain angle for the composite. Both appear to deviate from the optical isoclinic parameter by equal amounts.

Thus it is clear that the Mohr's circle of birefringence concept is successful in the interpretation of not only the isochromatics but also the isoclinics in orthotropic models.

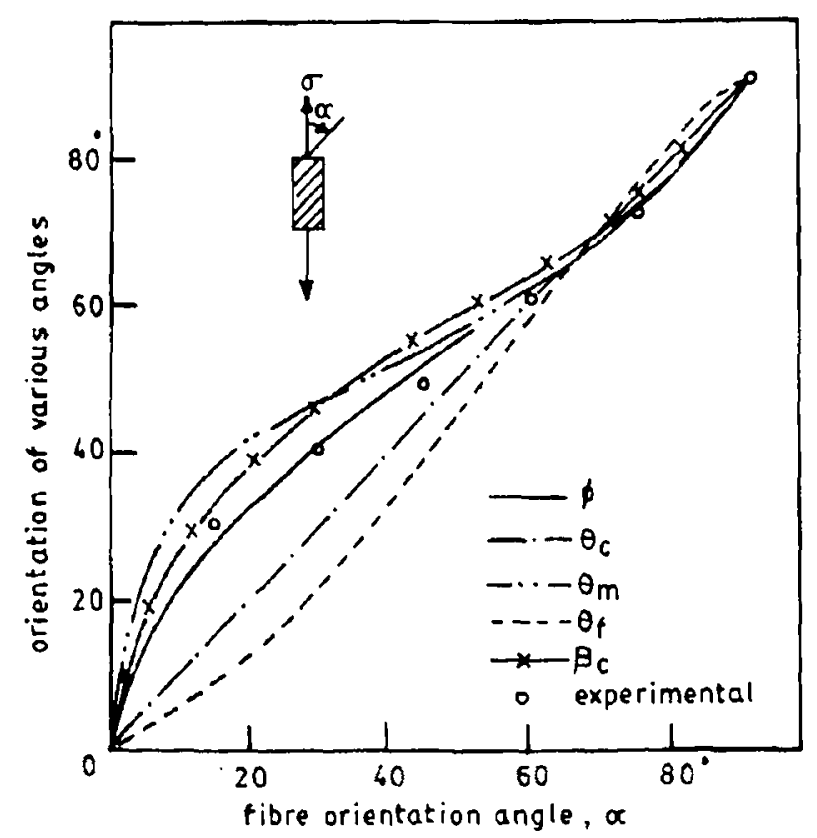

Figure 2. Graph showing the variation of different angles such as optical isoclinic, principal stress direction of fibre, matrix and composite, and principal strain direction of the composite as a function of fibre orientation angle (from Prabhakaran 1976). 


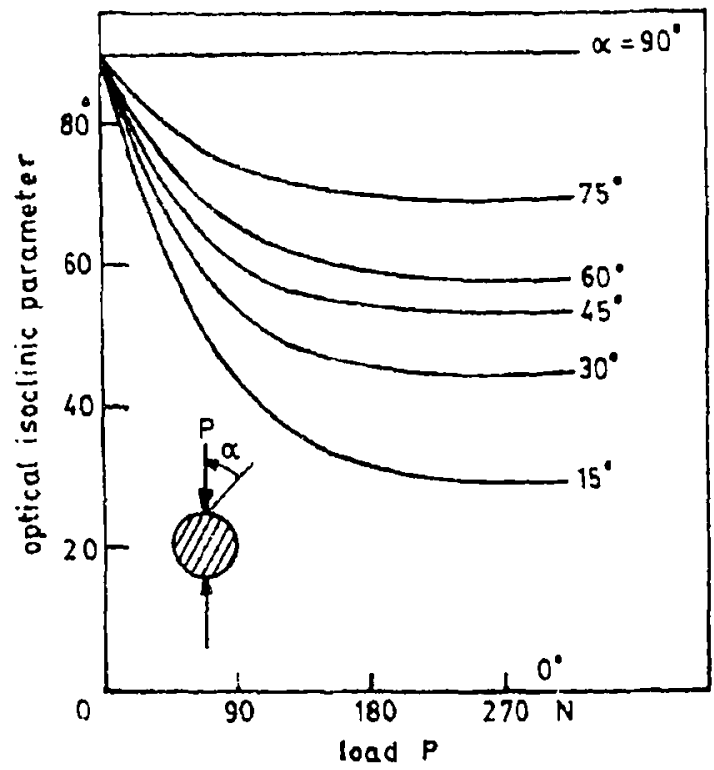

Figure 3. Optical isoclinic parameter (measured at the centre of the disc) as a function of load with fibre orientation angle as a parameter (from Prabhakaran 1976).

\section{Calibration of photo-orthotropic composites}

The development of the theory of birefringence of anisotropic composites has proceeded mainly along the following direction; stress-strain models, analogy, integrated photoelasticity and tensorial nature of birefringence. All these studies have concluded that three independent photoelastic constants are needed to characterize orthotropic model material.

Interrelationships between stress and strain optic coefficients have been given in (i2) to (17). It is more difficult to determine strain-fringe values experimentally as compared to stress-fringe values. This is due to the fact that application of uniaxial stress is easier than application of uniaxial strain.

Using tension specimens with fibres oriented at $0^{\circ}$ and $90^{\circ}$, it is easy to determine $f_{L}$ and $f_{T}$. On the other hand, determination of $f_{L T}$ is not easy since it requires

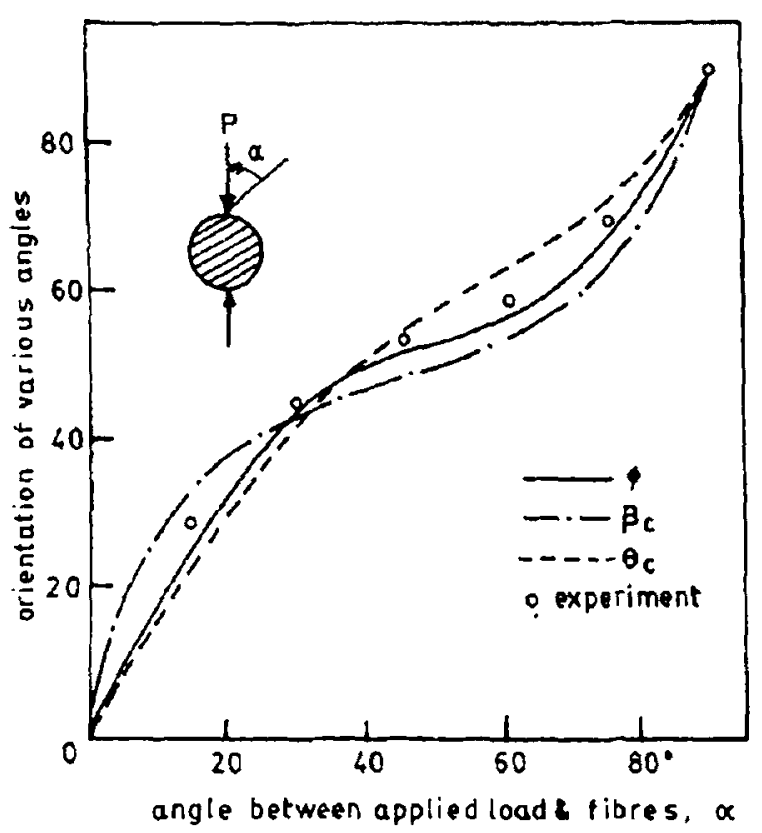

Figure 4. Graph showing the variation of various angles such as optical isoclinic, principal stress and strain directions at the centre of the disc as a function of fibre orientation angle (from Prabhakaran 1976). 
loading the specimen in pure shear. One can adopt anyone of the following three approaches depending on the accuracy desired.

(1) One approach is to use the interrelationship obtained in (18) if $V_{f}$ is less than $6 \%$.

(2) An approximate method to determine $f_{L T}$ is by obtaining $f_{\pi / 4}$ initially, by conducting a $45^{\circ}$ off-axis coupon test and then using this value in the following relation to get $f_{L T}$.

$$
f_{L T} / f_{\pi / 4}=\left[1+(1 / 4)\left\{f_{L T} / f_{L}\right\}^{2}\left\{1-\left(f_{L} / f_{T}\right)\right\}^{2}\right]^{1 / 2}
$$

The error in equating $f_{L T}$ to $f_{\pi / 4}$ depends upon the ratios, $f_{L T} / f_{L}$ and $f_{L} / f_{T}$. This error is small (usually less than $3 \%$ ).

(3) An exact method for finding $f_{L T}$ has been reported by Chaturvedi (1978) and is as follows.

It is seen from (3) that $f_{\alpha}$ is directly related to $f_{L r}$ when

$$
f_{T} / f_{L}=\tan ^{2} \alpha_{0}
$$

The values of $x_{0}$ can be determined by knowing $f_{L}$ and $f_{T}$ of the composite. If the unidirectional composite is subjected to the $\alpha_{0}{ }^{\circ}$ coupon test, then

$$
f_{L T}=f_{\alpha 0} \cdot \sin 2 \alpha_{0}
$$

Strain-fringe values can be determined by using the interrelationships between stress-fringe and strain-fringe values $[(12)$ to $(17)]$.

In the case of isotropic materials, accurate determination of $f_{0}$ is very critical since this is the only parameter which relates theoretical and experimental results. Following the same approach, it is essential that $f_{L}, f_{T}$ and $f_{L T}$ are determined with extreme care. In the determination of $f_{L T}$, the second method is widcly used. However, the method proposed by Chaturvedi $(1978)$ is better and is recommended for cases where higher accuracies are demanded.

\section{Influence of residual birefringence}

Curing of all types of epoxy or polyester resins is accompanied by a change of volume. Due to this shrinkage, residual birefringence is introduced. This is unavoidable while fabricating a transparent composite for conducting photo-orthotropic experiments. It has been found experimentally (Pipes \& Dalley 1973) that the principal direction of residual birefringence is identical with the direction of the fibres.

In the literature, accounting for residual birefringence is basically done in two different ways. In one approach, the final fringe order and optical isoclinic angle are expressed as functions of residual birefringence. Cernosek (1975) using integrated photoelasticity and Mittal (1975) based on Toupin's (1963) theory of deformable dielectrics obtained identical expressions for fringe order and isoclinic angle incorporating the effect of residual birefringence. The total fringe order $N_{T}$ in the presence of residual birefringence is given by

$$
N_{T}=t \cdot\left[\left[\left(N_{P} / t\right)+\left(\sigma_{L} / f_{L}\right)-\left(\sigma_{T} / f_{T}\right)\right]^{2}+\left[\left(2 \tau_{L T} / f_{L T}\right)\right]^{2}\right]^{1 / 2}
$$


and the isoclinic angle $\phi_{T}$ in the presence of residual stress is given by

$$
\tan 2 \phi_{T}=\left(2 \tau_{L T} / f_{L T}\right) /\left[\left(N_{r} / t\right)+\left(\sigma_{L} / f_{L}\right)-\left(\sigma_{T} / f_{T}\right)\right]
$$

Using the above expression for $\phi_{T}$ Cernosek (1975) was able to fit the experimental results of Pipes \& Dalley (1973) very precisely. The above expressions are strictly valid only for cases in which residual birefringence is in the direction of fibres.

The other approach is to extract the load-induced birefringence and the isoclinic angle. Cernosek (1975) has shown that an orthotropic composite can be modelled as a retarder without any rotation. Due to this, extraction of initial birefringence is very similar to the procedure adopted in isotropic cases (Guenter 1977). This has been shown using the tensorial nature of birefringence by Knight \& Pih (1976). Load induced birefringence is given by

and

$$
N=\left[N_{T}^{2}+N_{r}^{2}-2 N_{T} N_{r} \cos 2\left(\phi_{T}-\phi_{r}\right)\right]^{1 / 2},
$$

$$
\tan 2 \phi=\left(N_{T} \cdot \sin 2 \phi_{T}-N_{r} \cdot \sin 2 \phi_{r}\right) /\left(N_{T} \cdot \cos 2 \phi_{T}-N_{r} \cdot \cos 2 \phi_{r}\right),
$$

$\phi_{T}$ and $\phi_{r}$ should be referred to the $L$-axis of the composite. Cernosek (1975) and Mittal (1975) assumed $\phi_{r}$ to be 0 . This has been experimentally confirmed by Pipes and Dalley (1973).

When a theoretical reconstruction of fringes is required (which is gradually acquiring significance), one can use (22) and (23) (Ccrnosek 1975; Mittal 1975). On the other hand, (24) and (25) are appropriate for experimental calculations.

\section{Conclusions}

Birefringent isotropic model materials can be characterized by a single photoelastic constant. However, three constants are required for the complete characterization of photo-orthotropic model materials. In general, the directions of principal stresses and strains are not identical. Hence, the stress-optic laws and the strain-optic laws are different for these model materials. Both stress-optic and strain-optic theories highlight the need for three independent calibration constants for photo-orthotropic materials. Interrelationships do exist between stress-optic and strain-optic coefficients.

It can be concluded that when an accurate photoelastic analysis of an orthotropic model is needed, either the stress-optic law or the strain-optic law can be employed. Both of them are equally complex to use. When an approximate analysis is sufficient, the approximate strain-optic law proposed by Agarwal (1983) can be employed. This approximate strain-optic law is, however, exact for a balanced composite.

Due to complexities in the birefringent phenomena in orthotropic model materials, the isochromatic fringes observed in such materials are neither representative of contours of principal stress differences nor of the principal strain differences. Further, the optical isoclinics seen neither represent the principal stress directions nor the principal strain directions.

In view of the above, separation of principal stresses or strains is not a simple task even for a two-dimensional problem. Several methods have been proposed by various investigators.

Sampson (1970) proposed that the components of birefringence according to the Mohr's circle of birefringence, along with the differential equations of equilibrium, 
could be used in a shear difference method. Another method to separate the principal stresses has been addressed by Agarwal \& Jha (1983). Recently, Prabhakaran (1982) has summarized various techniques for separating principal stresses.

The research work reported in this paper is supported in part by an Indo-US project jointly funded by NSF of USA (Grant No. INT-90011089), and the Department of Science and Technology, India and an ARDB project (no. 540) of the Government of India.

\section{List of symbols}

$E_{L}, E_{T} \quad$ Young's modulus of composite in $L$ and $T$ directions;

$f_{\alpha} \quad$ material stress fringe value of composite for a fibre orientation angle

$f_{L}, f_{T}, f_{L T}$

$f_{\varepsilon I,}, f_{z T}, f_{\varepsilon L T}$ $\alpha$;

$G_{L T}$

$n$

$N$

$N_{r}$

$N_{T}$

$p, q$

$t$

$V_{s}$

$\alpha$

$\beta_{c}$

$2 \delta_{S}$

$2 \delta_{m}$

$2 \delta_{0}$

$\varepsilon_{p}, \varepsilon_{q}$ material stress fringe values of composite in $L, T$ and $L T$ directions; material strain fringe values of composite in $L, T$ and $L T$ directions; shear modulus of the composite w.r.t. $L-T$ axes; total numbers of fibres traversed by light; load induced birefringence in the composite; residual birefringence in the composite; total observed birefringence in the composite; principal stresses in the composite; thickness of the model material; volume fraction of fibre in composite; orientation of $L$-axis of the composite w.r.t. $Y$-axis; principal strain direction in composite w.r.t. $L$-axis; relative phase retardation in a single fibre; relative retardation in matrix surrounding one fibre; total retardation in a composite laminate; principal strains in the composite;

$\varepsilon_{L}, \varepsilon_{T}, \gamma_{L T}$ strain components referred to $L-T$ axis;

$\Theta_{c}, \Theta_{f}, \Theta_{m} \quad$ principal stress directions in composite, fibre, and matrix, respectively, w.r.t. $L$ axis;

$v_{i j} \quad$ Poisson's ratio of composite w.r.t. $(i, j)$ axes;

$\sigma_{L}, \sigma_{T}, \tau_{L T} \quad$ stress components referred to $L-T$ axis;

$\phi$ load induced optical isoclinic angle w.r.t. $L$-axis;

$\phi_{r}$ residual isoclinic angle in the composite w.r.t. $L$ axis;

$\phi_{T}$ total observed isoclinic angle in the composite w.r.t. $L$ axis;

$\Omega \quad \Theta_{m}-\Theta_{f}$.

\section{References}

Aben H K 1966 Optical phenomena in photoelastic models by the rotation of principal axes. Exp. Mech. 6: 13-22

Agarwal B D 1983 An approximate method of orthotropic photoelastic analysis. Exp. Mech. 23: $55-58$

Agarwal B D, Chaturvedi S K 1970 Improved birefringent composites and assessment of photoelastic theories. Fiber Sci. Technol. 11: 399-412 
Agarwal B D, Chaturvedi S K 1982 Exact and approximate strain optic laws for orthotropic photoelastic materials. Fiber Sci. Technol. 33: 146-150

Agarwal B D, Jha P K 1983 A stress separation technique for photo-orthotropic elasticity. Compos. Technol. Rev. 5: 84-88

Agarwal B D, Tiwari N, Ramesh K 1991 Development and characterization of transparent composite Jaminates. In Second Annual Progress Report on the ARDB project on Application of Image Processing Technique to the determination of S.I.F. in orthotropic composite materials, Indian Institute of Technology, Kanpur, pp. 19-45

Bert C W 1972 Theory of photoelasticity for birefringent filamentary composites. Fiber Sci. Technol. 5: 165-171

Bhagavantham S 1966 Crystal symmetry and physical properties of crystals (New York: Academic Press)

Cernosek J 1975 On the photoelastic response of composites. Exp. Mech. 15(9): 354-357

Chaturvadi S K 1978 Some experimental stress analysis techniques for fibrous composites, $\mathrm{Ph} D$ thesis, Indian Institute of Technology, Kanpur

Chaturvedi S K 1982 Fundamental concepts of photoelasticity for anisotropic composite materials. Lett. Appl. Eng. Sci. 20(1): 145-157

Dally J W, Alfirevich I 1963 Application of birefringent coatings to glass-fiber-reinforced plastics. Exp. Mech. 9: 97-102

Dally J W, Prabhakaran R 1971 Photo-orthotropic elasticity. Exp. Mech. 11: 346-356

Doyle J F 1978 A unified approach to optical constitutive relations. Exp. Mech. 18:416-420

Doyle J F 1980 Constitutive relations in photomechanics. Int. J. Mech. Sci. 22: 1-8

Grakh I I, Mozhenskaya A F 1971 A type of mechanically anisotropic optically sensitive bodies. Mekhanika Polimerov 5: $835-839$

Greszczuk L B 1966 Theoretical and experimental studies on properties and behaviour of filamentary composites. Proc. 21st Annual Tech. Conf., SPI Reinforced Plastics Div. (Washington, DC: Soc. Plastics Ind.) Section 8-A

Guenter F 1977 Superposition of photoelastic data. Exp. Mech. 17(1): 33-36

Hayashi T 1962 Photoelastic method of experimentation for stress analysis in orthotropic structures. Proc. Fourth Int. Symp. Space Technol. Science, Tokyo pp. 156-169

Horridge G A 1955 A polarized light study of glass fiber laminates. Br. J. Appl. Phys. 6: 314-319

Jha P K 1980 An approximate theory of photo orthotropic-elasticity, M Tech thesis; Indian Institute of Technology, Kanpur

Knight C E, Pih H 1976 Orthotropic stress-optic law for plane stress photoelasticity of composite materials. Fibre Sci. Technol. 9: 297-313

Mittal R K 1975 On the residual birefringence in anisotropic photoelastic materials. Strain 11: $55-57$

Netrebko V P 1971 Optical polarization method of studying the stress state of anisotropic bodies. Mekhanika Tverdogo Tela 1: 94-100

Pih H, Knight C E 1969 Photoelastic analysis of anisotropic fiber reinforced composites. $J$. Comp. Mater. 3: 94-107

Pipes R B, Dally J W 1972 On the birefringent coating method of stress analysis for fiber reinforced laminates. Exp. Mech. 12: 272-277

Pipes R B, Dalley J W 1973 On the fiber-reinforced birefringent composite materials. Exp. Mech. i3(8): 348--349

Pipes R B, Rose J L 1974 Strain-optic law for a certain class of birefringent composite. Exp. Mech. 14: $355-360$

Pockels F 1906 Lehrbuch der Kristalloptik (Leipzig: B G Teubner)

Prabhakaran R 1975 A strain-optic law for orthotropic model materials. AIAA J. 13: 723 728

Prabhakaran R 1976 The interpretation of isoclinics in photo-orthotropic elasticity. Exp. Mech. 16(1): 6-10

Prabhakaran R 1979 Developments in photo-orthotropic elasticity. Proc. IUTAM (Amsterdam: Sijthoft \& Noordhoff) pp. 559-581

Prabhakaran R 1980a Discussion of paper by Doyle. Exp. Mech. 20: 178-180

Prabhakaran R 1980b Fabrication of birefringent anisotropic model materials. Exp. Mech. 20: 320-321

Prabhakaran R 1982 Photo-orthotropic elasticity: A new technique for stress analysis of composites. Opt. Eng. 21: 679-688 
Ramesh K, Tiwari N 1991 Photo-orthotropic elasticity theories - a review, ARDB Report no. ARBD-STR-TR-91-540-01, Indian Institute of Technology, Kanpur

Sampson R C 1970 A stress-optic law for photoelastic analysis of orthotropic composites. Exp. Mech. 10: $210-215$

Toupin R A 1963 A dynamic theory of elastic dielectrics. Int. J. Eng. Sci. 1: 101

Tiwari N 1990 Development of transparent composite laminates and evaluation of Mode I stress intensity factor using photo-orthotropic elasticity, $M$ Tech thesis, Indian Institute of Technology, Kanpur

Whitney J M, Daniel Issac M, Byron Pipes R 1984 Experimental mechanics of fiber reinforced materials (Englewood Cliffs, NJ: Prentice Hall) 